\title{
Some Properties for Adjustable Entropy Function Method and Its Application to Power Systems Dynamic Economic Dispatch with Wind Integrated
}

\author{
Yaming Ren \\ College of Mechanical and Control Engineering, Guilin University of Technology, Guilin, China \\ Correspondence should be addressed to Yaming Ren; renyaming1981@gmail.com
}

Received 22 September 2021; Accepted 5 November 2021; Published 22 November 2021

Academic Editor: Jose R. C. Piqueira

Copyright (C) 2021 Yaming Ren. This is an open access article distributed under the Creative Commons Attribution License, which permits unrestricted use, distribution, and reproduction in any medium, provided the original work is properly cited.

\begin{abstract}
With the continuous development of the world economy, the development and utilization of environmentally friendly and renewable energy have become the trend in many countries. In this paper, we study the dynamic economic dispatch with wind integrated. Firstly, we take advantage of the positive and negative spinning reserve to deal with wind power output prediction errors in order to establish a dynamic economic dispatch model of wind integrated. The existence of a min function makes the dynamic economic dispatch model nondifferentiable, which results in the inability to directly use the traditional mathematical methods based on gradient information to solve the model. Inspired by the aggregate function, we can easily transform the nondifferentiable model into a smooth model when parameter $p$ tends to infinity. However, the aggregate function will cause data overflow when $p$ tends to infinity. Then, for solving this problem, we take advantage of the adjustable entropy function method to replace of aggregate function method. In addition, we further discuss the adjustable entropy function method and point out that the solution generated by the adjustable entropy function method can effectively approximate the solution of the original problem without parameter $p$ tending to infinity. Finally, simulation experiments are given, and the simulation results prove the effectiveness and correctness of the adjustable entropy function method.
\end{abstract}

\section{Introduction}

With the continuous development of the world economy, the development and utilization of environmentally friendly and renewable energy have become the trend in many countries. China has included building a clean, low-carbon, safe, and efficient modern energy system in its 13th five-year plan. In 2003, China's first million-kilowatt wind power base was built in Zhangiiakou and provided green and clean energy for the 2008 Beijing Olympic Games. After that, China has approved the establishment of Zhangjiakou as a renewable energy demonstration zone in 2015. In 2018, more than 10,000 new wind turbines were installed in China accounting for more than half of the total newly installed wind turbines in the world. With the increasing scale of wind power integration, people not only can enjoy the green energy provided by wind power but also can face the challenge brought by wind power to the stable operation of the power grid due to the inherent randomness, volatility, and intermittency of wind power.

In the actual power system, renewable energy resources such as wind power, solar power plant, geothermal power plant, and traditional petrochemical energy form a multiple energy system $[1,2]$. Therefore, more attention has been paid to the scheduling strategy considering the multienergy coordination in the field of electric power control. Akram et al. proposed a hybrid renewable energy and energy storage system comprised a battery energy storage system and a supercapacitor energy storage system [3]. To be specific, the energy storage system is used to realize peak load shifting for power system operation. Xu and Zhang reduced the impact of wind power fluctuations on the grid by coordinating solar power and wind resources [4]. Specifically, solar power converts the Sun's energy into heat energy, which is used to 
drive a generator to produce electricity. In the case that the actual output of wind power is greater than the expected output, the surplus electric energy can be stored in the form of heat energy. Correspondingly, when the actual output of wind power is less than the expected output, the thermal energy can be converted into electric energy to reduce the error between the actual and expected outputs of wind power. Electromechanical or electromagnetic storage such as batteries can achieve the same function but are more expensive than thermal energy storage devices, so it is more economical to use thermal energy storage. At the same time, researchers in China have applied the idea of multienergy coordination to practical power projects. The Republic Photovoltaic Park and LongYangXia hydropower station with an installed capacity of $850,000 \mathrm{~kW}$ are the world's first water-light complementary projects. ZhangBei national wind-solar storage and transportation demonstration base is the largest renewable energy base in the world that integrates wind power, photovoltaic power generation, energy storage, and power transmission, achieving the goal of smooth output, peak clipping, valley filling, and frequency control. In general, the multienergy coordinated dispatching strategy can effectively absorb the output of wind power and photovoltaic power station. However, the use of the multienergy coordinated scheduling strategy needs to meet certain conditions. To be more precise, the use of thermal energy storage requires special natural conditions, and the cost must be taken into account when using electromechanical or electromagnetic storage such as batteries as storage devices.

In addition to the multienergy coordination approach, another effective way to deal with the uncertainty of the generation output is to utilize the spinning reserve. In the process of power system operation, the power balance of the power system is destroyed due to power system equipment failures such as generator set malfunction, transmission line short circuit, and transform fault; we can use the spinning reserve to readjust the output of the generator set in order to restore the power system to a new power balance [5]. If we consider the inherent randomness, volatility, and intermittency of renewable energy such as wind power as a failure of the power system, then we can use the spinning reserve to deal with the inherent randomness, volatility, and intermittency of renewable energy. The advantage of the spinning reserve approach over the multienergy coordinated approach is that no additional storage facilities are required. Compared with the multienergy coordination method, the disadvantage of the spinning reserve approach is that the absorption capacity of the new energy is weak.

In the actual network of power systems, the method of multienergy coordination and the method of spinning reserve exist simultaneously. Both methods deal with the system power imbalance caused by the uncertainty of new energy output or other power failures. In this paper, the dynamic economic dispatch problem of the power system with large-scale wind power grids is studied from the perspective of spinning reserve.

\section{Mathematical Model of Dynamic Economic Dispatch}

2.1. Classical Power System Economic Dispatching Model. The classical power system economic dispatch model takes the generation cost as the objective function and considers two constraints: the active power balance constraint and the upper and lower limit constraint of the output of the generator set. The specific mathematical model is shown as follows [6]:

$$
\begin{aligned}
& \min F=\sum_{i=1}^{N} F\left(P_{i}\right)=\sum_{i=1}^{N}\left(a_{i} P_{i}^{2}+b_{i} P_{i}+c_{i}\right) \\
& \text { s.t } \sum_{i=1}^{N} P_{i}=P_{D} \\
& P_{i, \min } \leq P_{i} \leq P_{i, \max },
\end{aligned}
$$

where the generation cost of the $i$-th generator set can be expressed as a quadratic function of its active power output $P_{i}$ and the parameters of the quadratic function of the $i$-th generator set are $a_{i}, b_{i}$, and $c_{i}$. $P_{D}$ represents the total system load, $P_{i, \text { min }}$ represents lower limits constraint of $i$-th generator set, and $P_{i, \max }$ represents upper limits constraint of $i$ th generator set.

\subsection{The Ideal Dynamic Economic Dispatching Model of Power} System without considering Fluctuation. The classical economic dispatching model of the power system can be regarded as a special case of the dynamic dispatching model of the power system: only a time section is considered. In other words, the power system dynamic economic scheduling problem is composed of a series of classical power system scheduling problems with coupling relations. The coupling relation is called the ramp rate limits constraint, and the corresponding mathematical expression is shown in the following formulas [7]:

$$
\begin{aligned}
& P_{i, h}-P_{i, h-1} \leq r_{u}^{i} \times T_{60}, \\
& P_{i, h-1}-P_{i, h} \leq r_{d}^{i} \times T_{60},
\end{aligned}
$$

where $P_{i, h}$ is the active power output of $i$-th unit at time $h$ and $r_{u}^{i}$ and $r_{d}^{i}$ are the upper and lower ramp rate limits, respectively. $T_{60}$ represents an operating cycle of a power system in minutes, $T_{60}=60$ minutes. A series of coupled power system economic scheduling problems together constitute dynamic economic scheduling problems, and the corresponding mathematical model is shown as follows: 


$$
\begin{gathered}
\min F=\sum_{h=1}^{H} \sum_{i=1}^{N} F\left(P_{i, h}\right)=\sum_{h=1}^{H} \sum_{i=1}^{N}\left(a_{i} P_{i, h}^{2}+b_{i} P_{i, h}+c_{i}\right) \\
\sum_{i=1}^{N} P_{i, h}+P_{w, h}=P_{D, h}, h=1,2, \ldots, H \\
\text { s.t } P_{i, \min } \leq P_{i, h} \leq P_{i, \max }, h=1,2, \ldots, H \\
\quad P_{i, h}-P_{i, h-1} \leq r_{u}^{i} \times T_{60}, h=2, \ldots, H \\
P_{i, h-1}-P_{i, h} \leq r_{d}^{i} \times T_{60}, h=2, \ldots, H,
\end{gathered}
$$

where $H$ represents the number of periods. $N$ Represents the number of generating sets. The other symbols have the same meaning as the symbols in formulas (1)-(3).

\subsection{The Mathematical Model for Dynamic Economic Dispatch} of Power System considering Wind Output Fluctuation. According to the constraint conditions of problem equation (4), it can be seen that $P_{i, h}$ satisfies $P_{i, h} \leq P_{i, \max }$ when $h=1$ and $P_{i, h}$ satisfies both $P_{i, h} \leq P_{i, \max }$ and $P_{i, h} \leq r_{u}^{i} \times T_{60}+P_{i, h-1}$ when $h=2, \ldots, H$. Therefore, we can get $P_{i, h} \leq P_{i, \max }$ when $h=1$ and $P_{i, h} \leq \min \left(r_{u}^{i} \times T_{60}+P_{i, h-1}, P_{i, \max }\right)$ when $h=$ $2, \ldots, H$. On the premise that the output error of wind power is ignored, the positive spinning reserve for $P_{i, h}$ can be expressed as $P_{i, \max }-P_{i, h}$ when $h=1$ and $\min \left(P_{i, \max }\right.$, $\left.P_{i, h-1}+r_{u}^{i} \times T_{60}\right)-P_{i, h}$ when $h=2, \ldots, H$. In practice, the actual active power output of wind power is not equal to the predicted value of wind power output. The system needs to adjust the output of the generator set to restore the active power balance within the specified time (10 minutes) [8]. Based on the above analysis, the positive spinning reserve for $P_{i, h}$ can be expressed as $\min \left(P_{i, h}-P_{i, \min }, r_{d}^{i} \times T_{10}\right)$ when $h=$ 1 and $\sum_{i=1}^{N} \min \left(\min \left(P_{i, \max }, P_{i, h-1}+r_{u}^{i} \times T_{60}\right)-P_{i, h}\right.$, $\left.r_{u}^{i} \times T_{10}\right)$ when $h=2, \ldots, H$. Similarly, we can get the negative spinning reserve for $P_{i, h}$. Let $P_{w, h} \times L_{u} \%$ and $P_{w, h} \times$ $L_{D} \%$ represent the maximum positive and negative prediction errors of wind power output, respectively. The spinning reserve constraint can be expressed as follows:

$$
\begin{gathered}
\sum_{i=1}^{N} \min \left(\min \left(P_{i, \max }, P_{i, h-1}+r_{u}^{i} \times T_{60}\right)-P_{i, h}, r_{u}^{i} \times T_{10}\right) \geq P_{w, h} \times L_{u} \%, \quad h=2, \ldots, H, \\
\sum_{i=1}^{N} \min \left(P_{i, h}-\max \left(P_{i, \min }, P_{i, h-1}-r_{d}^{i} \times T_{60}\right), r_{d}^{i} \times T_{10}\right) \geq P_{w, h} \times L_{D} \%, \quad h=2, \ldots, H, \\
\sum_{i=1}^{N} \min \left(P_{i, \max }-P_{i, h}, r_{u}^{i} \times T_{10}\right) \geq P_{w, h} \times L_{u} \%, \quad h=1, \\
\sum_{i=1}^{N} \min \left(P_{i, h}-P_{i, \min }, r_{d}^{i} \times T_{10}\right) \geq P_{w, h} \times L_{D} \%, \quad h=1 .
\end{gathered}
$$

By observing equations (5a) and (5b), we find that these equations contain two min functions and can be rewritten with only one min function as follows:

$$
\begin{aligned}
& \sum_{i=1}^{N} \min \left(P_{i, \max }-P_{i, h}, P_{i, h-1}+r_{u}^{i} \times T_{60}-P_{i, h}, r_{u}^{i} \times T_{10}\right) \geq P_{w, h} \times L_{u} \%, \quad h=2, \ldots, H, \\
& \sum_{i=1}^{N} \min \left(P_{i, h}-P_{i, \min }, P_{i, h}-P_{i, h-1}+r_{d}^{i} \times T_{60}, r_{d}^{i} \times T_{10}\right) \geq P_{w, h} \times L_{D} \%, \quad h=2, \ldots, H .
\end{aligned}
$$

Therefore, the dynamic economic dispatching model of power system considering wind output fluctuation can be written as follows: 


$$
\begin{aligned}
\min F= & \sum_{h=1}^{H} \sum_{i=1}^{N} F\left(P_{i, h}\right)=\sum_{h=1}^{H} \sum_{i=1}^{N}\left(a_{i} P_{i, h}^{2}+b_{i} P_{i, h}+c_{i}\right) \\
& \sum_{i=1}^{N} P_{i, h}+P_{w, h}=P_{D, h} \\
& P_{i, \min } \leq P_{i, h} \leq P_{i, \max } \\
& P_{i, h}-P_{i, h-1} \leq r_{u}^{i} \times T_{60} \\
& P_{i, h-1}-P_{i, h} \leq r_{d}^{i} \times T_{60} \\
& \sum_{i=1}^{N} \min \left(P_{i, \max }-P_{i, h}, r_{u}^{i} \times T_{10}\right) \geq P_{w, h} \times L_{u} \%, \quad h=1 \\
& \sum_{i=1}^{N} \min \left(P_{i, h}-P_{i, \min }, r_{d}^{i} \times T_{10}\right) \geq P_{w, h} \times L_{D} \%, \quad h=1 \\
& \sum_{i=1}^{N} \min \left(P_{i, \max }-P_{i, h}, P_{i, h-1}+r_{u}^{i} \times T_{60}-P_{i, h}, r_{u}^{i} \times T_{10}\right) \geq P_{w, h} \times L_{u} \%, \quad h=2, \ldots, H \\
& \sum_{i=1}^{N} \min \left(P_{i, h}-P_{i, \min }, P_{i, h}-P_{i, h-1}+r_{d}^{i} \times T_{60}, r_{d}^{i} \times T_{10}\right) \geq P_{w, h} \times L_{D} \%, \quad h=2, \ldots, H .
\end{aligned}
$$

\section{Smoothing Method for Dynamic Economic Dispatch}

3.1. Aggregate Function Method. It can be found that the constraint condition of the optimization model (equation (7)) contains the min function. Therefore, it is impossible to solve the problem equation (7) directly by using the traditional mathematical programming method based on gradient information. The intelligent evolution method does not require the objective function and constraint conditions to have some strict mathematical properties and can effectively deal with the optimization problems containing logical judgment conditions (such as min function). Kalakova et al. proposed a novel genetic algorithm to solve dynamic economic scheduling problems, during which the spinning reserve is expressed as min function [9]. Jiang et al. use a novel improved particle swarm optimization to solve dynamic economic scheduling problems, and the spinning reserve constraint contains the min function [10]. The advantage of an intelligent evolutionary algorithm is that the optimization problem does not need to have convex, smooth, differentiable, and other strict mathematical properties. At the same time, we cannot guarantee that the result obtained by the evolutionary algorithm is the optimal solution to the optimization problem because there is no effective judgment condition.

Another effective way to solve the problem equation (7) is to smooth the min function and transform the problem equation (7) into a traditional convex optimization problem. In fact, the min function can be easily converted to an equivalent $\max$ function, $\max \left\{f_{i}(x)\right\}=-\min \left\{-f_{i}(x)\right\} . \quad$ Fortunately, Xingsi proposed an aggregate function to realize the smoothing of max function [11]. After that, an aggregate function has been widely used in many fields. Reference [12] combines the aggregate function with the unscented Kalman filter to solve the GPS/inertial navigation system integration. In reference [13], an aggregate function has been applied to solve the Min-Max problem. In reference [14], an aggregate function has been used for solving the inequalityconstrained least-squares problem.

For the following convex problem,

$$
\begin{aligned}
& \min f(x) \\
& \text { subject to } \begin{array}{l}
\gamma(x) \leq 0 \\
\gamma(x)=\max \left\{g_{i}(x)\right\} .
\end{array}
\end{aligned}
$$

Theorem 1 (see [11]). If p tends to infinity, problem equation (8) is equivalent to the following problem:

minimize $f(x)$

$$
g_{p}(x) \leq 0
$$

subject to

$$
g_{p}(x)=\left(\frac{1}{p}\right) \ln \left\{\sum_{i=1}^{m} \exp \left[p g_{i}(x)\right]\right\} \text {. }
$$

Xingsi further pointed out that [11]

$$
0 \leq g_{p}(x)-\gamma(x) \leq \frac{\ln (m)}{p},
$$

where $m$ is the number of $g_{i}(x)$. It can be known that $g_{p}(x)-\gamma(x)$ lies in the interval $[0,(\ln (m) / p)]$. 
3.2. Adjustable Entropy Function Method. According to the description of Theorem 1, we can find that if $p$ tends to infinity, problem equation (9) is equivalent to problem equation (8). $g_{p}(x)$ is called the aggregate function. Experimental results in references [15-17] show that the aggregate function method is valuable in the application. However, the aggregate function will cause data overflow when $p$ tends to infinity. The case of the aggregate function reminds us of the external penalty function method. When using the external penalty function method deals with the constraints of convex optimization problems, only the penalty parameter tends to infinity, and the solution obtained by the external penalty function method is the optimal solution of the original problem. However, when the penalty function tends to infinity, it is easy to cause the ill of unconstrained optimization problems after transformation [18]. Similarly, if the traditional mathematical optimization algorithm based on gradient information is used to solve the problem equation (9), the Hessian matrix of unconstrained the problem equation (9) after transformation is likely to be an ill-conditioned matrix when $p$ tends to infinity, resulting in the termination of the calculation. In order to overcome this shortcoming, Qingzhi et al. proposed an adjustable entropy function for solving Max-Min problem $\min \max _{1 \leq i \leq m}\left\{g_{i}(x)\right\}$ [19]. After that, Qing et al. take advantage of the adjustable entropy function to solve the support vector machine [20].

For max function

$$
\gamma(x)=\max _{1 \leq i \leq m}\left\{g_{i}(x)\right\} .
$$

The adjustable entropy function can be expressed as follows [19]:

$$
\begin{gathered}
f_{p}(x, \mu)=\left(\frac{1}{p}\right) \ln \left\{\sum_{i=1}^{m} \mu_{i} \exp \left[p g_{i}(x)\right]\right\}, \\
\mu_{i} \geq 0, \sum_{i=1}^{m} \mu_{i}=1,
\end{gathered}
$$

where $\mu_{i}$ represents the adjustable factor.

According to the description of equations (12) and (13), it is possible for any $\exp \left[p g_{i}(x)\right]$ to be $\exp \left[p \max \left\{g_{i}(x)\right\}\right]$ in initial state, and the corresponding parameter $u_{i}$ is the probability of $\exp \left[p g_{i}(x)\right]$ being $\exp \left[p \max \left\{g_{i}(x)\right\}\right]$. Therefore, we set the initial value of the adjustable factor as $\mu_{i}^{0}=(1 / m)$. The updating strategy of adjustable factor can be described as follows:

$$
\mu_{i}^{k}=\frac{\mu_{i}^{k-1} \exp \left[p g_{i}\left(x^{k}\right)\right]}{\sum_{i=1}^{m} \mu_{i}^{k-1} \exp \left[p g_{i}\left(x^{k}\right)\right]} .
$$

Then, the iterative strategy based on the adjustable entropy function method (AEFM) for solving problem equation (8) can be expressed as follows:
Step 1. For $\forall p>0$, setting the number of iterations $k=0$, the maximum number of iterations is set as $k_{\max }$; the initial point for $x$ is set as $x^{0}$; and the initial value of the adjustable factor is set as follows:

$$
\mu_{i}^{0}=\frac{1}{m} \text {. }
$$

Step 2. The initial point for $x$ is set as $x^{k}$, and $x^{k+1}$ is obtained by solving

$\min f(x)$

$$
f_{p}\left(x, \mu^{k}\right) \leq 0
$$

subject to

$$
f_{p}\left(x, \mu^{k}\right)=\left(\frac{1}{p}\right) \ln \left\{\sum_{i=1}^{m} \mu_{i}^{k} \exp \left[p g_{i}(x)\right]\right\} .
$$

Step 3. Check the stop criterion. If

$$
\max \left(\left|x^{k}-x^{k-1}\right|\right)<\varepsilon
$$

then stop. If not, go to step 4 .

Step 4. Updating adjustable factor,

$$
\mu_{i}^{k+1}=\frac{\mu_{i}^{k} \exp \left[p g_{i}\left(x^{k+1}\right)\right]}{\sum_{j=1}^{m} \mu_{i}^{k} \exp \left[p g_{i}\left(x^{k+1}\right)\right]} .
$$

Step 5. If $k>k_{\max }$, the iteration fails to convergence. If $k<k_{\max }, k=k+1$ go to step 2 .

Qingzhi et al. proposed the iterative strategy based on the adjustable entropy function method (AEFM), and some numerical simulation experiments show that AEFM iterative scheme is convergent [19]. However, Qingzhi et al. did not theoretically prove that the solution obtained by adjusting the entropy iteration strategy is equivalent to the solution of problem equation (8). In this paper, we further discuss AEFM iterative scheme and give some new properties for AEFM iterative scheme.

(1) For $f_{p}(x, \mu)$, if $g_{i}(x)$ is convex and the dimension of $x$ is one, then $f_{p}(x, \mu)$ is convex.

(2) For $\forall p>0$, sequence $\left\{x^{k}\right\}$ is generated by the AEFM iterative scheme. If sequence $\left\{x^{k}\right\}$ is convergence $\lim x^{k}=x^{*}$, then we can get that $x^{*}$ is the optimal $\stackrel{K}{K}$ solution of the original problem.

\subsection{Some Properties for AEFM}

Theorem 2. For $\forall p>0$ 


$$
\left(\frac{1}{p}\right) \ln \left(\sum_{i \in M} \mu_{i}\right) \leq f_{p}(x, \mu)-\gamma(x) \leq 0, \quad M=\left\{j \mid g_{j}(x)-\max _{1 \leq j \leq m}\left\{g_{j}(x)\right\}=0\right\} .
$$

Proof.

$$
\begin{aligned}
f_{p}(x, \mu)-\gamma(x) & =\left(\frac{1}{p}\right) \ln \left\{\sum_{i=1}^{m} \mu_{i} \exp \left[p g_{i}(x)\right]\right\}-\max \left\{g_{i}(x)\right\} \\
& =\left(\frac{1}{p}\right) \ln \left\{\sum_{i=1}^{m} \mu_{i} \exp \left[p g_{i}(x)\right]\right\}-\left(\frac{1}{p}\right) \ln \left\{\exp \left[p \max \left\{g_{i}(x)\right\}\right]\right\} \\
& =\left(\frac{1}{p}\right) \ln \left\{\frac{\sum_{i=1}^{m} \mu_{i} \exp \left[p g_{i}(x)\right]}{\left.\exp \left[p \max \left\{g_{i}(x)\right\}\right]\right\}}\right. \\
& =\left(\frac{1}{p}\right) \ln \left\{\sum_{i=1}^{m} \mu_{i} \exp \left[p g_{i}(x)-p \max \left\{g_{i}(x)\right\}\right]\right\} .
\end{aligned}
$$

In fact, there must be a set $M$ that satisfies

$M=\left\{j \mid g_{j}(x)-\max \left\{g_{j}(x)\right\}=0, \quad j=1,2, \ldots, m\right\}$.

It is clear that

$$
\begin{aligned}
& p\left(g_{i}(x)-\max \left\{g_{i}(x)\right\}\right) \leq 0 \\
& \Rightarrow 0<\exp \left[p\left(g_{i}(x)-\operatorname{tmaxn}\left\{g_{i}(x)\right\}\right)\right] \leq 1 .
\end{aligned}
$$

According to equation (22), we can get

$f_{p}(x, \mu)-\gamma(x)$

$$
\begin{aligned}
& =\left(\frac{1}{p}\right) \ln \left(\sum_{i=1}^{m} \mu_{i} \exp \left[p\left(g_{i}(x)-\max \left\{g_{i}(x)\right\}\right)\right]\right) \\
& \leq\left(\frac{1}{p}\right) \ln \left(\sum_{i=1}^{m} \mu_{i}\right) \\
& =0 .
\end{aligned}
$$

It is clear that

$$
\begin{aligned}
f_{p}(x, \mu)-\gamma(x) & =\left(\frac{1}{p}\right) \ln \left(\sum_{i=1}^{m} \mu_{i} \exp \left[p\left(g_{i}(x)-\max \left\{g_{i}(x)\right\}\right)\right]\right) \\
& \geq\left(\frac{1}{p}\right) \ln \left(\sum_{i \in M} \mu_{i} \exp \left[p\left(g_{i}(x)-\max \left\{g_{i}(x)\right\}\right)\right]\right) \\
& =\left(\frac{1}{p}\right) \ln \left(\sum_{i \in M} \mu_{i}\right) .
\end{aligned}
$$

Theorem 2 is proved.

Theorem 3. For $f_{p}(x, \mu)$, if $g_{i}(x)$ is convex and the dimension of $x$ is one, then $f_{p}(x, \mu)$ is convex.

Proof.

$$
f_{p}^{\prime}(x, \mu)=\frac{\sum_{i=1}^{m} \mu_{i} g_{i}^{\prime}(x) \exp \left[p g_{i}(x)\right]}{\sum_{i=1}^{m} \mu_{i} \exp \left[p g_{i}(x)\right]}
$$

$f_{p}^{\prime \prime}(x, \mu)$

$=-\frac{p \sum_{i=1}^{m} \mu_{i} g_{i}^{\prime}(x) \exp \left[p g_{i}(x)\right]}{\left(\sum_{i=1}^{m} \mu_{i} \exp \left[p g_{i}(x)\right]\right)^{2}} \sum_{i=1}^{m} \mu_{i} g_{i}^{\prime}(x) \exp \left[p g_{i}(x)\right]+\frac{\sum_{i=1}^{m} \mu_{i} g_{i}^{\prime \prime}(x) \exp \left[p g_{i}(x)\right]+p \sum_{i=1}^{m} \mu_{i} g_{i}^{\prime}(x) g_{i}^{\prime}(x) \exp \left[p g_{i}(x)\right]}{\sum_{i=1}^{m} \mu_{i} \exp \left[p g_{i}(x)\right]}$ 
It is clear that

$$
\begin{aligned}
& p\left(\sum_{i=1}^{m} \mu_{i} g_{i}^{\prime}(x) \exp \left[p g_{i}(x)\right]\right)\left(\sum_{i=1}^{m} \mu_{i} g_{i}^{\prime}(x) \exp \left[p g_{i}(x)\right]\right) \\
& =p \sum_{i=1}^{m}\left(\mu_{i} g_{i}^{\prime}(x) \exp \left[p g_{i}(x)\right]\right)^{2}+2 p \sum_{i=1}^{m} \sum_{j=i+1}^{m}\left(\mu_{i} g_{i}^{\prime}(x) \exp \left[p g_{i}(x)\right]\right)\left(\mu_{j} g_{j}^{\prime}(x) \exp \left[p g_{j}(x)\right]\right)
\end{aligned}
$$

and

$$
\begin{aligned}
& p\left(\sum_{i=1}^{m} \mu_{i} g_{i}^{\prime}(x) g_{i}^{\prime}(x) \exp \left[p g_{i}(x)\right]\right)\left(\sum_{i=1}^{m} \mu_{i} \exp \left[p g_{i}(x)\right]\right) \\
& =p \sum_{i=1}^{m}\left(\mu_{i} g_{i}^{\prime}(x) \exp \left[p g_{i}(x)\right]\right)^{2}+p \sum_{i=1}^{m} \sum_{j=i+1}^{m}\left(\mu_{i} g_{i}^{\prime}(x) g_{i}^{\prime}(x) \exp \left[p g_{i}(x)\right]\right)\left(\mu_{j} \exp \left[p g_{j}(x)\right]\right) \\
& \quad+p \sum_{j=1}^{m} \sum_{i=j+1}^{m}\left(\mu_{i} g_{i}^{\prime}(x) g_{i}^{\prime}(x) \exp \left[p g_{i}(x)\right]\right)\left(\mu_{j} \exp \left[p g_{j}(x)\right]\right)
\end{aligned}
$$

Combining equation (13) and equations (26)-(28), we can get

$$
\begin{aligned}
& f_{p}^{\prime \prime}(x, \mu) \\
& =\frac{\left(\sum_{i=1}^{m} \mu_{i} g_{i}^{\prime \prime}(x) \exp \left[p g_{i}(x)\right]\right)\left(\sum_{i=1}^{m} \mu_{i} \exp \left[p g_{i}(x)\right]\right)+p \sum_{i=1}^{m} \sum_{j=i+1}^{m}\left(\mu_{i} \exp \left[p g_{i}(x)\right]\right)\left(\mu_{j} \exp \left[p g_{j}(x)\right]\right)\left(g_{i}^{\prime}(x)-g_{j}^{\prime}(x)\right)^{2}}{\left(\sum_{i=1}^{m} \mu_{i} \exp \left[p g_{i}(x)\right]\right)^{2}} \\
& \Rightarrow f_{p}^{\prime \prime}(x) \geq \frac{\left(\sum_{i=1}^{m} \mu_{i} g_{i}^{\prime \prime}(x) \exp \left[p g_{i}(x)\right]\right)\left(\sum_{i=1}^{m} \mu_{i} \exp \left[p g_{i}(x)\right]\right)}{\left(\sum_{i=1}^{m} \mu_{i} \exp \left[p g_{i}(x)\right]\right)^{2}}
\end{aligned}
$$

Because $g_{i}(x)$ is convex, we can get

$$
g_{i}^{\prime \prime}(x) \geq 0 \text {. }
$$

Combining equation (13) and equations (29) and (30), we can get

$$
f_{p}^{\prime \prime}(x, \mu) \geq 0 \text {. }
$$

Because the second derivative of $f_{p}(x, \mu)$ is greater than or equal to zero, $f_{p}(x, \mu)$ is convex.

Theorem 3 is proved.
Theorem 4. If sequence $\left\{x^{k}\right\}$ is generated by the AEFM iterative scheme, then we can get

$$
u_{i}^{k}=\frac{\prod_{1 \leq n \leq k} \exp \left(p g_{i}\left(x^{n}\right)\right)}{\sum_{i=1}^{m} \prod_{1 \leq n \leq k} \exp \left(p g_{i}\left(x^{n}\right)\right)}
$$

Proof:. According to the description of AEFM iterative scheme, the initial value of the adjustable factor as $\mu_{i}=(1 / m)$, then we can get

$$
u_{i}^{1}=\frac{(1 / m) \exp \left(p g_{i}\left(x^{1}\right)\right)}{\sum_{i=1}^{m}(1 / m) \exp \left(p g_{i}\left(x^{1}\right)\right)}=\frac{\exp \left(p g_{i}\left(x^{1}\right)\right)}{\sum_{i=1}^{m} \exp \left(p g_{i}\left(x^{1}\right)\right)}=\frac{\prod_{1 \leq n \leq 1} \exp \left(p g_{i}\left(x^{n}\right)\right)}{\sum_{i=1}^{m} \prod_{1 \leq n \leq 1} \exp \left(p g_{i}\left(x^{n}\right)\right)}
$$


Assuming the conclusion equation (32) holds when $k=s-1$, we can get

$$
u_{i}^{s}=\frac{u_{i}^{s-1} \exp \left(p g_{i}\left(x^{s}\right)\right)}{\sum_{i=1}^{m} u_{i}^{s-1} \exp \left(p g_{i}\left(x^{s}\right)\right)}=\frac{\left(\prod_{1 \leq n \leq s-1} \exp \left(p g_{i}\left(x^{n}\right)\right) / \sum_{i=1}^{m} \prod_{1 \leq n \leq s-1} \exp \left(p g_{i}\left(x^{n}\right)\right)\right) \exp \left(p g_{i}\left(x^{s}\right)\right)}{\sum_{i=1}^{m}\left(\prod_{1 \leq n \leq s-1} \exp \left(p g_{i}\left(x^{n}\right)\right) / \sum_{i=1}^{m} \prod_{1 \leq n \leq s-1} \exp \left(p g_{i}\left(x^{n}\right)\right)\right) \exp \left(p g_{i}\left(x^{s}\right)\right)}=\frac{\prod_{1 \leq n \leq s} \exp \left(p g_{i}\left(x^{n}\right)\right)}{\sum_{i=1}^{m}\left\{\prod_{1 \leq n \leq s} \exp \left(p g_{i}\left(x^{n}\right)\right)\right\}^{\prime}}
$$

Theorem 4 is proved.

Theorem 5. For $\forall p>0$, sequence $\left\{x^{k}\right\}$ and $\left\{\mu^{k}\right\}$ are generated by the AEFM iterative scheme. If sequence $\left\{x^{k}\right\}$ is convergence $\lim _{K \longrightarrow \infty} x^{k}=x^{*}$, then we can get

$$
\begin{aligned}
& \lim _{K \longrightarrow \infty} u_{i \in Q\left(x^{*}\right)}^{k}=0, \\
& \lim _{K \longrightarrow \infty} \sum_{i \in M\left(x^{*}\right)} u_{i}^{k}=1, \\
& Q(x)=\left\{j \mid g_{j}(x)<\max \left\{g_{j}(x)\right\}, \quad j=1,2, \ldots, m\right\}, \\
& M(x)=\left\{j \mid g_{j}(x)-\max \left\{g_{j}(x)\right\}=0, \quad j=1,2, \ldots, m\right\} .
\end{aligned}
$$

Proof:. According to the description of Theorem 4, we can get

$$
\begin{aligned}
\frac{u_{i \in Q\left(x^{*}\right)}^{k}}{\sum_{i \in M\left(x^{*}\right)} u_{i}^{k}} & =\frac{\prod_{1 \leq n \leq k} \exp \left(p g_{i \in Q\left(x^{*}\right)}\left(x^{n}\right)\right)}{\sum_{i \in M\left(x^{*}\right)} \prod_{1 \leq n \leq k} \exp \left(p g_{i}\left(x^{n}\right)\right)} \\
& =\frac{\left(\prod_{1 \leq n \leq k} \exp \left(p g_{i \in Q\left(x^{*}\right)}\left(x^{n}\right)\right) / \prod_{1 \leq n \leq k} \exp \left(p g_{i \in M\left(x^{*}\right)}\left(x^{n}\right)\right)\right)}{\left(\sum_{i \in M\left(x^{*}\right)} \prod_{1 \leq n \leq k} \exp \left(p g_{i}\left(x^{n}\right)\right) / \prod_{1 \leq n \leq k} \exp \left(p g_{i \in M\left(x^{*}\right)}\left(x^{n}\right)\right)\right)} \\
& =\frac{\prod_{1 \leq n \leq k}\left(\exp \left(p g_{i \in Q\left(x^{*}\right)}\left(x^{n}\right)\right) / \exp \left(p g_{i \in M\left(x^{*}\right)}\left(x^{n}\right)\right)\right)}{\left(\sum_{i \in M\left(x^{*}\right)} \prod_{1 \leq n \leq k} \exp \left(p g_{i}\left(x^{n}\right)\right) / \prod_{1 \leq n \leq k} \exp \left(p g_{i \in M\left(x^{*}\right)}\left(x^{n}\right)\right)\right)}
\end{aligned}
$$

It is clear that

$$
\begin{aligned}
& \sum_{i \in M\left(x^{*}\right)} \prod_{1 \leq n \leq k} \exp \left(p g_{i}\left(x^{n}\right)\right) \geq \prod_{1 \leq n \leq k} \exp \left(p g_{i \in M\left(x^{*}\right)}\left(x^{n}\right)\right)>0 \\
& \Rightarrow \sum_{i \in M\left(x^{*}\right)} \frac{\prod_{1 \leq n \leq k} \exp \left(p g_{i}\left(x^{n}\right)\right)}{\prod_{1 \leq n \leq k} \exp \left(p g_{i \in M\left(x^{*}\right)}\left(x^{n}\right)\right) \geq 1}
\end{aligned}
$$

Because sequence $\left\{x^{k}\right\}$ is convergence $\lim _{K \longrightarrow \infty} x^{k}=x^{*}$, there must be a positive integer $N$ that satisfies

$$
\begin{aligned}
& \left|\lim _{k \longrightarrow \infty}\left(g_{i \in Q\left(x^{*}\right)}\left(x^{k}\right)-g_{i \in Q\left(x^{*}\right)}\left(x^{*}\right)\right) \leq \varepsilon 1\right|, \quad k>N \text { and } \varepsilon 1>0, \\
& \left|\lim _{k \longrightarrow \infty}\left(g_{i \in M\left(x^{*}\right)}\left(x^{k}\right)-g_{i \in M\left(x^{*}\right)}\left(x^{*}\right)\right) \leq \varepsilon 2\right|, \quad k>N \text { and } \varepsilon 2>0, \\
& \left|\lim _{k \longrightarrow \infty}\left(g_{i \in Q\left(x^{*}\right)}\left(x^{*}\right)-g_{i \in M(x *)}\left(x^{*}\right)\right) \geq \varepsilon 1+\varepsilon 2\right|, \quad k>N, \varepsilon 1>0, \varepsilon 2>0 .
\end{aligned}
$$


Based on equations (38)-(40), we can get

$$
g_{i \in Q\left(x^{*}\right)}\left(x^{k}\right)-g_{i \in M(x *)}\left(x^{k}\right)<0, \quad k>N .
$$

Based on equation (41), we can get

$$
\begin{aligned}
0 & <\frac{\exp \left(p g_{i \in Q\left(x^{*}\right)}\left(x^{n}\right)\right)}{\exp \left(p g_{i \in M\left(x^{*}\right)}\left(x^{n}\right)\right)}\langle 1, k\rangle N \\
& \Rightarrow \lim _{k \longrightarrow \infty} \prod_{N+1 \leq n \leq k} \frac{\exp \left(p g_{i \in Q\left(x^{*}\right)}\left(x^{n}\right)\right)}{\exp \left(p g_{i \in M\left(x^{*}\right)}\left(x^{n}\right)\right)}=0 \\
& \Rightarrow \lim _{k \rightarrow \infty} \prod_{1 \leq n \leq k} \frac{\exp \left(p g_{i \in Q\left(x^{*}\right)}\left(x^{n}\right)\right)}{\exp \left(p g_{i \in M\left(x^{*}\right)}\left(x^{n}\right)\right)}=0 .
\end{aligned}
$$

Combining equations (37) and (42), we can get

$$
\lim _{k \longrightarrow \infty} \frac{u_{i \in Q\left(x^{*}\right)}^{k}}{\sum_{i \in M\left(x^{*}\right)} u_{i}^{k}}=0 .
$$

Combining equations (13) and (43), we can get

$$
\begin{aligned}
& \lim _{K \longrightarrow \infty} \sum_{i \in M\left(x^{*}\right)} u_{i}^{k}=1, \\
& \lim _{K \longrightarrow \infty} u_{i \in Q\left(x^{*}\right)}^{k}=0 .
\end{aligned}
$$

Theorem 5 is proved.

Theorem 6. For $\forall p>0$, sequences $\left\{x^{k}\right\}$ and $\left\{\mu^{k}\right\}$ are generated by the AEFM iterative scheme. For $f_{p}(x, \mu)$, on the premise that variable $x$ is a one-dimensional variable. If sequence $\left\{x^{k}\right\}$ is convergence $\lim x^{k}=x^{*}$, then we can get that $x^{*}$ is the optimal solution of problem equation (8).

Proof. According to Theorem 2, we can get

$$
\lim _{k \rightarrow \infty}\left(\frac{1}{p}\right) \ln \left(\sum_{i \in M\left(x^{k}\right)} u_{i}^{k}\right)=0 .
$$

According to Theorem 2 and squeeze theorem, we can get

$$
\lim _{k \rightarrow \infty}\left(f_{p}\left(x^{k}, \mu^{k}\right)-\gamma\left(x^{k}\right)\right)=0
$$

According to the description of Theorem 3, it can be known that $f_{p}(x, \mu)$ is convex and problem equation (16) is convex optimization problems. Meanwhile, according to the description in equation (46), we can say that $x^{*}$ is the optimal solution of problem equation (8).

Theorem 6 is proved.

\section{Numerical Experiments}

4.1. Test System of 10-Unit System. In this paper, the 10-unit system is used as test system and all data from reference [21]. Reference [21] provides a 10-unit system with 24-period, and we take the data of the first 8 periods. The generator data are shown in Table 1, and the load data are shown in Table 2.
In addition, the fmincon function provided by MATLAB software is used to solve optimization problem, and the option for fmincon function is set as options = optimset(“Algorithm," "interior-point," "MaxIter," 50000, "MaxFunEvals," 50000).

In the actual power system, the standard deviation of the output error of wind power can be calculated through the historical output data of wind power. This paper mainly discusses the model of the dynamic economic dispatching model of power system involving large-scale wind power, the forecasting method of wind power output, and the statistical characteristics of historical wind power output error data that are not involved. In this paper, the wind power output forecast is given by a periodic function $150+50 \sin ((1: 8) * 2 * p i / 8)$, and the wind power output data is shown in Table 3. Meanwhile, we assume that the maximum wind power output error accounts for $30 \%$ of the predicted wind power output $\left(L_{u} \%=L_{D} \%=30 \%\right)$.

4.2. Simulation Analysis. In this paper, we employ two different strategies to solve the test system with different $p$ $(p=1,2, \ldots, 50)$.

(1) AFM: the aggregate function method

(2) AEFM: the adjustable entropy function method with $\varepsilon=10^{-3}$ and $k_{\max }=100$

In fact, the min function can be easily converted to an equivalent max function, $\max \left\{f_{i}(x)\right\}=-\min \left\{-f_{i}(x)\right\}$. We can obtain the positive and negative spinning reserve capacity of the system by substituting the optimal solution obtained by AFM into the left-hand side of inequality equations (5a)-(5d), denoted as PSR1 and NSR1. If we replace the left-hand side of inequality equations (5a)-(5d) by aggregate function, we can get the approximate positive and negative spinning reserve capacity of the system, denoted as APSR1 and ANSR1.

Similarly, we can obtain the positive and negative spinning reserve capacity of the system by substituting the optimal solution obtained by AEFM into the left-hand side of inequality equations (5a)-(5d), denoted as PSR2 and NSR2. If we replace the left-hand side of inequality equations (5a)-(5d) with the adjustable entropy function, we can get the approximate positive and negative spinning reserve capacity of the system, denoted as APSR2 and ANSR2.

If the optimal solution obtained by AFM is equivalent to the optimal solution to the original problem equation (7), then we can get PSR $1-$ APSR $1=0$ and NSR1 - ANSR $1=0$. That is to say |PSR1 - APSR1| and |NSR1 - ANSR1| can indirectly measure the proximity between the optimal solution obtained by AFM and the optimal solution of the original problem equation (7). The smaller |PSR1 - APSR1| and |NSR1 - ANSR1|, the better. Similarly, it is clear that |PSR2 - APSR2| and |NSR2 - ANSR2| can indirectly measure the proximity between the optimal solution obtained by AEFM and the optimal solution of the original problem equation (7).

Specific to the test system used in this paper, the dimension of |PSR1-APSR1|, |NSR1 - ANSR1|, 
TABle 1: The data for generator set.

\begin{tabular}{|c|c|c|c|c|c|c|c|}
\hline Unit & $P_{\max }(\mathrm{MW})$ & $P_{\min }(\mathrm{MW})$ & $a\left(\$ / \mathrm{MW}^{2} \mathrm{~h}\right)$ & $b$ (\$/MWh) & $c(\$ / h)$ & UR (MW/min) & $\mathrm{DR}(\mathrm{MW} / \mathrm{min})$ \\
\hline 1 & 470 & 150 & 0.00043 & 21.6 & 958.2 & 1.33 & 1.33 \\
\hline 2 & 460 & 135 & 0.00063 & 21.05 & 1313.6 & 1.33 & 1.33 \\
\hline 3 & 340 & 73 & 0.00039 & 20.81 & 604.07 & 1.33 & 1.33 \\
\hline 4 & 300 & 60 & 0.0007 & 23.9 & 471.6 & 0.83 & 0.83 \\
\hline 5 & 243 & 73 & 0.00079 & 21.620 & 480.29 & 0.83 & 0.83 \\
\hline 6 & 160 & 57 & 0.00056 & 17.87 & 601.75 & 0.83 & 0.83 \\
\hline 7 & 130 & 20 & 0.00211 & 16.51 & 502.7 & 0.50 & 0.50 \\
\hline 8 & 120 & 47 & 0.0048 & 23.23 & 639.4 & 0.50 & 0.50 \\
\hline 9 & 80 & 20 & 0.10908 & 19.58 & 455.6 & 0.50 & 0.50 \\
\hline 10 & 55 & 55 & 0.00951 & 22.54 & 692.4 & 0.50 & 0.50 \\
\hline
\end{tabular}

TABle 2: The load data.

\begin{tabular}{|c|c|c|c|c|c|c|c|c|}
\hline Period & 1 & 2 & 3 & 4 & 5 & 6 & 7 & 8 \\
\hline Load (MW) & 1,036 & 1,110 & 1,258 & 1,406 & 1,480 & 1,628 & 1,702 & 1,776 \\
\hline
\end{tabular}

TABLE 3: The wind power output data.

\begin{tabular}{lccccccc}
\hline Period & 1 & 2 & 3 & 4 & 5 & 6 & 7 \\
Pw (MW) & 185.35 & 200 & 185.35 & 150 & 114.64 & 100 & 114.64 \\
\hline
\end{tabular}
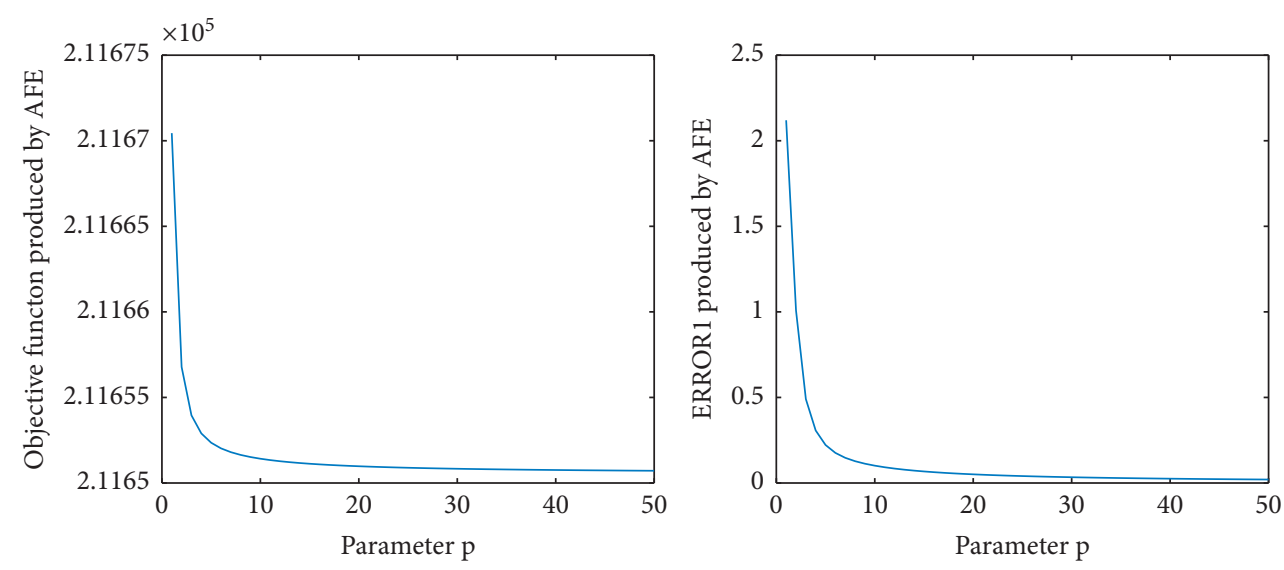

FIgURE 1: The trend of the objective function and ERROR1 produced by AFM with different $p$.

|PSR2 - APSR2|, and |NSR2 - ANSR2| are eight. In order to reflect the effect of the algorithm more simply and intuitively, we define ERROR1 = $\max (\mid$ PSR1 - APSR1|, |NSR1 ANSR1|)

and ERROR2 $=\max (\mid P S R 2-$ APSR2|, |NSR2 - ANSR2| $)$ to describe the approximation degree of AFM and AEFM to the original problem. The trend of the objective function and ERROR1 produced by AFM with different $p$ is shown in Figure 1. The trend of the objective function and ERROR2 produced by AEFM with different $p$ is shown in Figure 2 .

According to the data shown in Figure 1, the objective function and ERROR1 decrease gradually with the increase of parameter $p$. In the initial stage, the objective function and ERROR1 decrease rapidly. However, when the parameter $p$ is greater than 20, the objective function and ERROR1 do not decrease significantly with the increase of parameter $p$.
In practice, the fmincon function terminates due to data overflow when the parameter $p$ is greater than or equal to 56 . This is consistent with our analysis of AFM in Section 3. The objective function and ERROR1 are $2.1165 \times 10^{5}$ and 0.0196 when $p=55$. Therefore, we can say that AFM cannot accurately approximate the solution of the original problem, and the solution obtained by AFM is only a suboptimal solution of the original problem.

According to the information shown in Figure 2, the objective function and ERROR2 distribute in a small range. For the objective function, the difference between maximum and minimum is $6.6887 \times 10^{-4}$, and the average of the objective function is $2.1165 \times 10^{5}$. Therefore, we can say that the fluctuation of the objective function is negligible. For ERROR2, the maximum value of ERROR2 is $6.6961 \times 10^{-4}$, which is far less than the minimum of the ERROR2. 

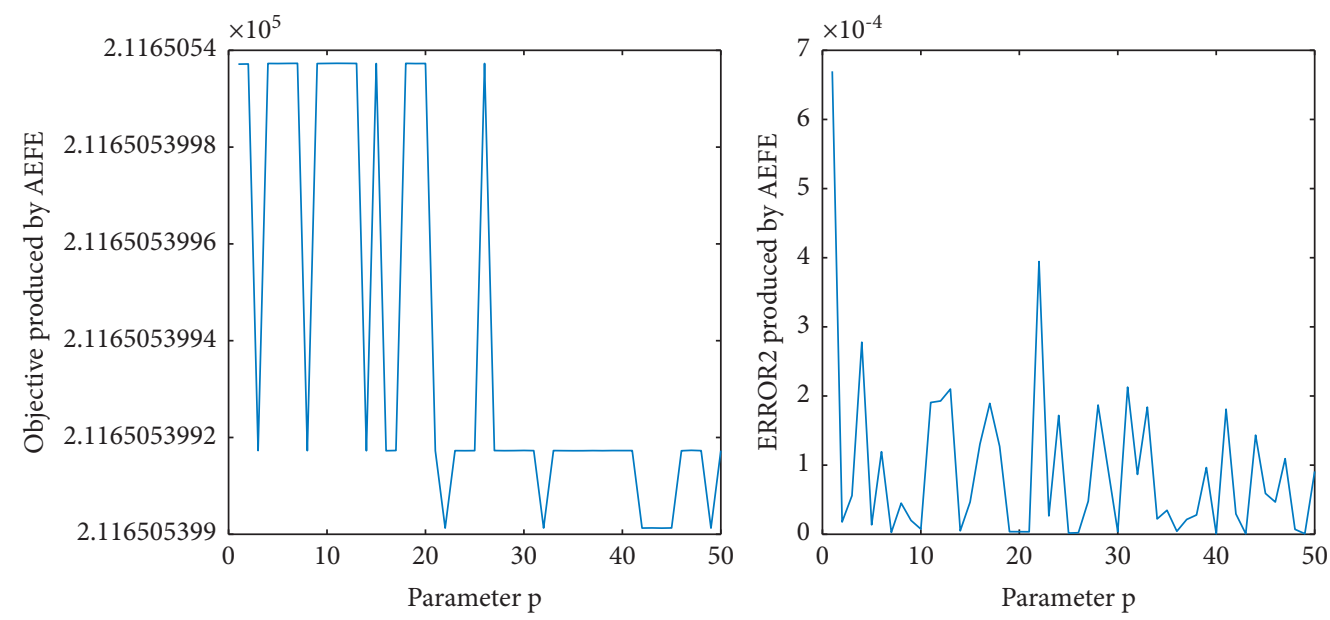

FIgURE 2: The trend of the objective function and ERROR2 produced by AEFM with different $p$.

Compared with AFM, the optimal solution obtained by AEFM can better approximate the solution of the original problem; the optimal solution obtained by AEFM is stable with different $p$; and AEFM is robust in terms of the choice of the parameter $p$.

\section{Conclusion}

In this paper, the dynamic economic dispatch model with wind integrated is studied. We take advantage of the positive and negative spinning reserve to deal with wind power output prediction error. Through the analysis of the mathematical model, it can be found that the dynamic economic dispatch model with wind integrated contains a min function. The existence of the min function makes the model nondifferentiable, which results in the inability to directly use the traditional mathematical methods based on gradient information to solve the model. Inspired by AFM, we can easily transform the nondifferentiable programming problem into a smooth programming problem when parameter $p$ tends to infinity. However, AFM will cause data overflow when $p$ tends to infinity. For solving this problem, we take advantage of AEFM to transform the nondifferentiable programming problem into a smooth programming problem. In addition, we further discuss AEFM and point out that the solution generated by AEFM can effectively approximate the solution of the original problem without parameter $p$ tending to infinity. Finally, the 10-unit system is employed as the test system, and the simulation results prove the effectiveness and correctness of AEFM.

\section{Data Availability}

The data in this paper were generated by MATLAB, and the MATLAB codes are available any time if needed from author Yaming Ren (renyaming1981@gmail.com).

\section{Conflicts of Interest}

The author declares that there are no conflicts of interest regarding the publication of this paper.

\section{Acknowledgments}

This work was supported by the Guangxi Science and Technology Base and Talent Special Project (grant number GuiKeAD20159077) and the Foundation of Guilin University of Technology (grant number GLUTQD2018001]).

\section{References}

[1] S. Dawn, P. K. Tiwari, and A. K. Goswami, "Efficient approach for establishing the economic and operating reliability via optimal coordination of wind-PSH-solar-storage hybrid plant in highly uncertain double auction competitive power market," IET Renewable Power Generation, vol. 12, no. 10, pp. 1189-1202, 2018.

[2] F. Mohamad, J. Teh, and H. Abunima, "Multi-objective optimization of solar/wind penetration in power generation systems," IEEE Access, vol. 7, pp. 169094-169106, 2019.

[3] U. Akram, M. Khalid, and S. Shafiq, "An innovative hybrid wind-solar and battery-supercapacitor microgrid systemdevelopment and optimization," IEEE Access, vol. 5, pp. 25897-25912, 2017.

[4] T. Xu and N. Zhang, "Coordinated operation of concentrated solar power and wind resources for the provision of energy and reserve services," IEEE Transactions on Power Systems, vol. 32, no. 2, pp. 1260-1271, 2017.

[5] K. Bruninx and E. Delarue, "Endogenous probabilistic reserve sizing and allocation in unit commitment models: cost-effective, reliable, and fast," IEEE Transactions on Power Systems, vol. 32, no. 4, pp. 2593-2603, 2017.

[6] Z. Wang, C. Shen, F. Liu, X. Wu, C.-C. Liu, and F. Gao, "Chance-constrained economic dispatch with non-Gaussian correlated wind power uncertainty," IEEE Transactions on Power Systems, vol. 32, no. 6, pp. 4880-4893, 2017.

[7] W. S. Cheng and H. F. Zhang, "A dynamic economic dispatch model incorporating wind power based on chance constrained programming," Energies, vol. 8, no. 1, pp. 233-256, 2015.

[8] N. Shouman, Y. G. Hegazy, and W. A. Omran, "Hybrid mean variance mapping optimization algorithm for solving stochastic based dynamic economic dispatch incorporating wind power uncertainty," Electric Power Components and Systems, vol. 48, no. 16-17, pp. 1786-1797, 2021. 
[9] A. Kalakova, H. S. V. S. K. Nunna, P. K. Jamwal, and S. Doolla, "A novel genetic algorithm based dynamic economic dispatch with short-term load forecasting," IEEE Transactions on Industry Applications, vol. 57, no. 3, pp. 2972-2982, 2021.

[10] W. Jiang, Z. Yan, and Z. Hu, "A novel improved particle swarm optimization approach for dynamic economic dispatch incorporating wind power," Electric Power Components and Systems, vol. 39, no. 5, pp. 461-477, 2011.

[11] L. Xingsi, "An aggregate constraint method for non-linear programming," Journal of the Operational Research Society, vol. 42, no. 11, pp. 1003-1010, 1991.

[12] H. Yu, Z. Li, J. Wang, and H. Han, "Data fusion for a GPS/INS tightly coupled positioning system with equality and inequality constraints using an aggregate constraint unscented Kalman filter," Journal of Spatial Science, vol. 65, no. 3, pp. 377-399, 2020.

[13] Y. Xiao and B. Yu, "A truncated aggregate smoothing Newton method for minimax problems," Applied Mathematics and Computation, vol. 216, no. 6, pp. 1868-1879, 2010.

[14] J. Peng, H. Zhang, S. Shong, and C. Guo, "An aggregate constraint method for inequality-constrained least squares problems," Journal of Geodesy, vol. 79, no. 12, pp. 705-713, 2006.

[15] X. Jiang, Y. Yang, Y. Lu, and M. Cao, "Flattened aggregate function method for nonlinear programming with many complicated constraints," Numerical Algorithms, vol. 86, no. 1, pp. 103-122, 2021.

[16] S. He, H. Zhang, and X. Li, "Solving frictional contact problems by two aggregate-function-based algorithms," Acta Mechanica Sinica, vol. 21, no. 5, pp. 467-471, 2005.

[17] H. Yang, J. Yan, and X. Li, "Solving inverse viscoelasticity problems via aggregate function approach," International Journal of Solids and Structures, vol. 40, no. 13-14, pp. 3707-3716, 2003.

[18] D. P. Bertsekas, "Multiplier methods: a survey," Automatica, vol. 12, no. 2, pp. 133-145, 1976.

[19] Y. Qingzhi, Y. DeZhuang, and Z. Minhong, "Adjustable entropy function method (in Chinese)," Mathematica Numerica Sinica, vol. 23, no. 1, pp. 81-86, 2001.

[20] Q. Wu, S. Liu, and L. Zhang, "Adjustable entropy function method for support vector machine," Journal of Systems Engineering and Electronics, vol. 19, no. 5, pp. 1029-1034, 2008.

[21] P. Attaviriyanupap, H. Kita, E. Tanaka, and J. Hasegawa, "A hybrid EP and SQP for dynamic economic dispatch with nonsmooth fuel cost function," IEEE Transactions on Power Systems, vol. 17, no. 2, pp. 411-416, 2002. 